\title{
A semiclassical approach to Coulomb scattering of conduction electrons on ionized impurities in nondegenerate semiconductors
}

\author{
N. A. Poklonski, ${ }^{\text {a) }}$ S. A. Vyrko, V. I. Yatskevich, ${ }^{\text {b) }}$ and A. A. Kocherzhenko \\ Belarusian State University, pr. F. Skarina 4, BLR-220050 Minsk, Republic of Belarus
}

(Received 13 December 2002; accepted 19 March 2003)

\begin{abstract}
In the proposed model of mobility, the time of electron-ion interaction equals the time taken by the conduction electron to pass a spherical region, corresponding to one impurity ion in crystal, and the minimum scattering angle is determined after Conwell-Weisskopf. We consider the acts of electron scattering on ions as independent and incompatible events. It is shown in the approximation of quasimomentum relaxation time, that for nondegenerate semiconductors, the mobility $\mu_{i}$, limited by the elastic scattering by impurity ions with the concentration $N_{i}$, is proportional to $T / N_{i}^{2 / 3}$; the Hall factor equals 1.4. The calculated dependences of the mobility of the majority charge carriers upon their concentration for different temperatures $T$ agree well with known experimental data. It is shown, that the Brooks-Herring formula $\mu_{\mathrm{BH}} \propto T^{3 / 2} / N_{i}$ gives overestimated values of mobility. Comparison of the calculations of mobility in degenerate semiconductors with experimental data also yields $\mu_{i}<\mu_{\mathrm{BH}}$. (C) 2003 American Institute of Physics. [DOI: 10.1063/1.1573735]
\end{abstract}

\section{INTRODUCTION}

The concentration of ionized impurities in crystal is usually evaluated from the comparison of experimental charge carrier mobility $\mu_{i}$, limited by the elastic scattering by ions, with the calculated $\mu_{\mathrm{BH}}$ by the Brooks-Herring (BH) formula (see, for example, Refs. 1-6). But, as noted in Ref. 7, the concentration of donors, found using $\mu_{\mathrm{BH}}$ from the Hall mobility of electrons in the $n$ part of the InSb ingot with a $p-n$ junction measured at $T=77 \mathrm{~K}$, is several times greater than those found from the distribution curve of donors along the ingot. The conclusion that $\mu_{i}<\mu_{\mathrm{BH}}$ is confirmed by the comparison of experimental data for different semiconductors with the calculations using the BH formula (see Ref. 8). The fact that $\mu_{i}<\mu_{\mathrm{BH}}$ is also confirmed by the simulation of the dependence of the drift mobility on the doping impurity concentration using the Monte Carlo method. ${ }^{9}$

In the $\mathrm{BH}$ model, the interaction time of the conduction electron with one impurity ion is nominally taken to be infinite. In the Conwell-Weisskopf $(\mathrm{CW})$ model $^{2-6,10}$ the minimum scattering angle (maximum impact parameter) is introduced. It is known, that in numerical simulation of the dependence of the drift velocity of the electrons on the electric-field strength in $n \mathrm{GaAs}^{11}$ the $\mathrm{CW}$ model gives better agreement with experiment than the BH model. When simulating the scattering by impurity ions in semiconductors by the Monte Carlo method, ${ }^{12}$ the Ridley approximation ${ }^{4}$ is used, which reconciles the $\mathrm{BH}$ and $\mathrm{CW}$ models by introducing the geometrical probability that the ion is the nearest scattering center for the conduction electron. Moreover, as pointed out in Ref. 6: “... it is difficult to observe experimentally a $\mu_{i} \propto T^{3 / 2}$ behavior over a wide temperature range."

\footnotetext{
${ }^{a}$ Electronic mail: poklonski@bsu.by

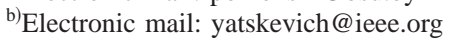

The purpose of this work is to calculate the electron (hole) mobility in relaxation time approximation under the condition, that the act of elastic pure Coulomb scattering by an ion continues only during the time taken by the conduction electron to pass a spherical region of the crystal, corresponding to one impurity ion, and the minimum scattering angle is defined in accordance with CW.

Let us consider the crystalline semiconductor of volume $V$, which contains impurity ions with concentration $N_{i}$. Thus, the number of ions in the crystal is $N_{i} V$. We will describe the electron in the effective-mass approximation by the wave function in the plane wave form $\psi_{k}$ $=V^{-1 / 2} \exp (i k r)$, where $\boldsymbol{k}$ is the quasiwave vector, $\boldsymbol{r}$ is the coordinate of the electron. In such a description, the electron plane wave interacts with all impurity ions of the crystal.

The transport relaxation time $\tau_{k}$ of the quasiwave vector $\boldsymbol{k}$ of the electron with the kinetic energy $E=(\hbar \boldsymbol{k})^{2} / 2 m$ for elastic, independent, and incompatible scattering on $N_{i} V$ impurity ions of the same type in the crystal is given by: ${ }^{2,3,13}$

$$
\begin{aligned}
\frac{1}{\tau_{k}} & =N_{i} V \sum_{k^{\prime}} W_{\boldsymbol{k}^{\prime} \boldsymbol{k}}(1-\cos \theta) \delta_{k^{\prime} k} \\
& =\frac{N_{i} V^{2}}{(2 \pi)^{3}} \int W_{\boldsymbol{k}^{\prime} \boldsymbol{k}}(1-\cos \theta) \delta\left(\frac{k^{\prime}-k}{k}\right) d^{3} \boldsymbol{k}^{\prime},
\end{aligned}
$$

where $N_{i}$ is the concentration of impurity ions, $W_{\boldsymbol{k}^{\prime} \boldsymbol{k}}$ is the electron scattering probability per unit time into the angle $\theta=\left(\widehat{\boldsymbol{k}, \boldsymbol{k}^{\prime}}\right)$ induced by the spherically symmetric Coulomb potential of an ion, $\delta_{k^{\prime} k}$ is the Kronecker delta, $\delta\left(\left(k^{\prime}\right.\right.$ $-k)(k)$ is the dimensionless Dirac delta function introduced to take into account the conservation law of quasiwave vector absolute value $\left(k^{\prime}=k\right)$ in elastic scattering, $\hbar=h / 2 \pi$ is Plank's constant, and $m$ is the electron effective mass.

In the first order of perturbation theory, the ratio of the probability of electron transition between $\boldsymbol{k}$ and $\boldsymbol{k}^{\prime}$ states 
with $E$ and $E^{\prime}$ energies to the "duration" $t_{c}$ of an impurity ion influence $U_{k^{\prime} k}$ upon the electron is equal: ${ }^{14}$

$$
W_{\boldsymbol{k}^{\prime} \boldsymbol{k}}=\frac{2}{t_{c}}\left|\frac{U_{\boldsymbol{k}^{\prime} \boldsymbol{k}}}{E^{\prime}-E}\right|^{2}\left\{1-\cos \left[\frac{t_{c}}{\hbar}\left(E^{\prime}-E\right)\right]\right\},
$$

when the probability of elastic scattering of electron by an ion $t_{c} W_{\boldsymbol{k}^{\prime} k} \ll 1$.

\section{MAIN IDEA OF THE PROPOSED MODEL}

Let us consider a doped semiconductor with impurity ions of one type in the charge state $Z \neq 0$. The first assumption is that the impurity ion with the charge $Z e$ does not possess internal degrees of freedom and scatters the electron with quasimomentum $\hbar \boldsymbol{k}$ elastically. The second assumption is that the conduction electron (regarded as a reasonably well-localized wave packet) interacts with one impurity ion only during the time $t_{c} \approx 2 R / v=2 R m / \hbar k$, where $v$ is the electron velocity, $R=\left(4 \pi N_{i} / 3\right)^{-1 / 3}$ is the radius of the spherical region of the crystal associated with an ion. ${ }^{15}$ After interaction with an ion during the time $t_{c}$, the electron scatters on the next ion over the same period of time and so on, i.e., scattering occurs permanently. ${ }^{16}$ Then, the minimum scattering angle $\theta_{i}$ of the electron on the Coulomb potential $Z e / 4 \pi \epsilon r$, where $\epsilon=\epsilon_{r} \epsilon_{0}$ is the static permittivity of a nondoped crystal, $\epsilon_{0}$ is the electric constant, and the duration $t_{c}=R(2 m / E)^{1 / 2}$ of the scattering act are bound up by a relation from classical mechanics: ${ }^{17}$

$$
\cot \frac{\theta_{i}}{2}=\frac{4 \pi \epsilon b m v^{2}}{|Z| e^{2}}=t_{c} \frac{4 \pi \epsilon}{|Z| e^{2}}\left(\frac{2}{m}\right)^{1 / 2} E^{3 / 2},
$$

where the maximum impact parameter $b=R$ is determined from the relation $4 \pi R^{3} / 3=1 / N_{i}$.

Note, that introduction of the finite interaction time $t_{c}$ of the electron with an impurity ion and minimum scattering angle $\theta_{i}$ makes the problem classical.

So, when determining the action sphere of an impurity ion upon the conduction electron, we follow the $\mathrm{CW}$ model, ${ }^{2-6,10}$ but additionally take into account the finiteness of time $t_{c}$ of the Coulomb interaction of the electron with an ion. The lower bound on the angle $\theta_{i}$ and upper on the time $t_{c}$ make consecutive scattering acts of the conduction electron by different impurity ions independent and incompatible events. ${ }^{18}$ According to Ref. 19, the condition of validity of classical mechanics while obtaining formula (3) is the minuteness of the electron de Broglie wavelength in comparison to the size of the scatterer: $2 \pi / k \ll 2 R$. It should be noted that at scattering, the form of the wave function does not change, only the direction of the electron quasiwave vector changes.

For the energy of Coulomb interaction of a conduction electron with an ion $U(r)=-Z e^{2} / 4 \pi \epsilon r$ we have: ${ }^{14}$

$$
U_{\boldsymbol{k}^{\prime} \boldsymbol{k}}=\int_{V} \psi_{\boldsymbol{k}^{\prime}}^{*} U(r) \psi_{\boldsymbol{k}} d^{3} \boldsymbol{r} \rightarrow U(k, \theta)=-\frac{Z e^{2}}{V \epsilon q^{2}},
$$

where $\hbar q=\hbar\left|\boldsymbol{k}^{\prime}-\boldsymbol{k}\right|=2 \hbar k \sin (\theta / 2)$ is the absolute value of electron quasimomentum transfer at elastic scattering $\left(\left|\boldsymbol{k}^{\prime}\right|=|\boldsymbol{k}|\right)$ on the angle $\theta ; \psi_{\boldsymbol{k}^{\prime}}^{*}=V^{-1 / 2} \exp \left(-i \boldsymbol{k}^{\prime} \boldsymbol{r}\right)$.
According to Ref. 14, the sufficient condition for the validity of formula (4) is $k R \gg 1$. The same condition, in Ref. 19 , denotes the range of validity for formula (3).

For $t_{c} \ll \hbar /\left|E^{\prime}-E\right|$, when in the scattering act on an impurity ion, the electron changes only the direction of motion, ${ }^{20}$ formula (2) is transformed according to Ref. 14 in the following way: ${ }^{21}$

$$
W_{\boldsymbol{k}^{\prime} \boldsymbol{k}} \rightarrow \frac{t_{c}}{\hbar^{2}}|U(k, \theta)|^{2}=\frac{2 R m}{\hbar^{3} k}|U(k, \theta)|^{2},
$$

where $U(k, \theta)$ is found from Eq. (4).

Substitution of Eq. (5) into Eq. (1) taking into account Eqs. (4) and (3) gives

$$
\frac{1}{\tau_{k}}=\left(\frac{3}{4 \pi}\right)^{1 / 3}\left(\frac{Z e^{2}}{4 \pi \epsilon}\right)^{2} \frac{N_{i}^{2 / 3}}{\hbar E} \ln \left[1+\left(\frac{8 \pi \epsilon R E}{|Z| e^{2}}\right)^{2}\right] .
$$

Note, that from Eqs. (2)-(5), the averaged by angles frequency $\nu_{i}$ of elastic collisions of the electron having the kinetic energy $E$ with ionized impurities can be written in the form:

$$
\begin{aligned}
\nu_{i} & =\frac{N_{i} V^{2}}{(2 \pi)^{3}} \int_{0}^{\infty} \int_{\theta_{i}}^{\pi} W_{k^{\prime} k} \delta\left(\frac{k^{\prime}-k}{k}\right) 2 \pi \sin \theta d \theta k^{\prime 2} d k^{\prime} \\
& =\frac{N_{i} R}{2 \hbar E}\left(\frac{Z e^{2}}{4 \pi \epsilon}\right)^{2} \cot ^{2} \frac{\theta_{i}}{2}=\frac{3}{2 \pi \hbar} E .
\end{aligned}
$$

The impurity scattering limited mobility of a conduction electron $\mu_{i}=e\langle\tau\rangle / m$ is determined by the average relaxation time $^{4-6,22}$

$$
\langle\tau\rangle=\int_{0}^{\infty} \tau_{k} E^{3 / 2} \frac{\partial f}{\partial E} d E\left(\int_{0}^{\infty} E^{3 / 2} \frac{\partial f}{\partial E} d E\right)^{-1},
$$

where $f=\left\{1+\exp \left[\left(E-E_{F}\right) / k_{B} T\right]\right\}^{-1}$ is the Fermi-Dirac function, $E_{F}$ is the Fermi energy, and $k_{B} T$ is the thermal energy.

The calculation of $\langle\tau\rangle$ for nondegenerate conduction electrons, when $f \approx \exp \left[\left(E_{F}-E\right) / k_{B} T\right]$, will be performed using the standard method. ${ }^{4-6,10}$ Namely, the function $\ln \left\{1+\left[8 \pi \epsilon R E /\left(|Z| e^{2}\right)\right]^{2}\right\}$ at energy $E=(\hbar k)^{2} / 2 m$ can be taken outside the integral in the numerator (7), when according to Eqs. (7) and (6), the expression $E^{5 / 2} \exp \left[\left(E_{F}-E\right) / k_{B} T\right]$ under the integral sign as a function of $E$ reaches its maximum value. This procedure corresponds to $E=5 k_{B} T / 2$. Then, as follows from formulas (6) and (7), the Hall factor $\left\langle\tau^{2}\right\rangle /\langle\tau\rangle^{2}=1.4$, and the mobility

$$
\mu_{i}=\frac{5}{2}\left(\frac{4 \pi}{3}\right)^{1 / 3} \frac{\hbar(4 \pi \epsilon)^{2} k_{B} T}{e^{3} m Z^{2} N_{i}^{2 / 3}} \frac{1}{\ln (1+\gamma)},
$$

where $\gamma=25\left[3 /\left(4 \pi N_{i}\right)\right]^{2 / 3}\left(4 \pi \epsilon k_{B} T\right)^{2} /\left(Z^{2} e^{4}\right)$.

Formula (8) is applicable to conduction electrons with the mean thermal energy $\left(\hbar k_{t}\right)^{2} / 2 m=3 k_{B} T / 2$ and wavelength $2 \pi / k_{t}$, when $k_{t} R>1$.

It should be noted here, that the value of the Hall factor $\left\langle\tau^{2}\right\rangle /\langle\tau\rangle^{2}=1.4$ obtained from Eq. (6) agrees more closely with experimental data for nondegenerate crystalline $p$ type semiconductors with prevailing scattering of holes by impurity ions, ${ }^{23,24}$ than $\left\langle\tau^{2}\right\rangle /\langle\tau\rangle^{2}=1.93$, which is obtained from $\mathrm{BH}$ and $\mathrm{CW}$ models. ${ }^{2,6}$ 
Let us consider the case, when in $n$-type crystal, the impurity ions of different types are presented, so that the electroneutrality equation takes the form: $n=\sum_{j} \Sigma_{z} Z N_{j z}$, where $n$ is the concentration of conduction electrons, and $N_{j z}$ is the concentration of ions of type $j$ in the charge state $Z(j)$. We are assuming that the minimum scattering angles $\theta_{i}$ for the impurities of type $j$ in the charge states $|Z(j)|>1$ and $|Z|=1$ are the same, i.e., $\theta_{i}(Z(j))=\theta_{i}(|Z|=1)$. Then, according to Eq. (3), for the maximum impact parameter $b_{j z}$ $=R_{j z}$ of electron scattering on the ion with the charge $Z(j) e$, we have $b_{j z}=R_{j z}=|Z(j)| R_{1}$, where $b_{1}=R_{1}$ is the maximum impact parameter for the ion in the charge state $|Z|=1$. The concentration of all impurity ions in the crystal $\Sigma_{j} \Sigma_{z \neq 0} N_{j z}$ satisfies the condition: $4 \pi / 3 \Sigma_{j} \Sigma_{z \neq 0} R_{j z}^{3} N_{j z}=1$, so that $\Sigma_{j} \Sigma_{z \neq 0}|Z|^{3} N_{j z}=3 /\left(4 \pi R_{1}^{3}\right)$. The time of interaction of the electron with an impurity ion (charge $Z e$ ) is $t_{c} \approx 2 R_{j z} / v$ $=2 R_{j z} m / \hbar k$, where $R_{j z}=|Z| R_{1}$ for all $j$ and $Z \neq 0$. In formulas (6) and (8), the presence of a different sort of ions may be considered by substituting:

$$
Z^{2} N_{i}^{2 / 3} \rightarrow\left(\sum_{j} \sum_{z} N_{j z}|Z|^{3}\right)^{2 / 3}
$$

It should be noted, that if the anisotropy of quasimomentum relaxation time in a multivalley semiconductor at elastic scattering on the ionized impurities can be neglected, then the mobility of electrons, say, in $n \mathrm{Si}$ is $\mu_{i}^{*}=e\langle\tau\rangle / m_{c}$ $=\left(m / m_{c}\right) \mu_{i}$, where $m=m_{d} / 6^{2 / 3}=0.36 m_{0}$ and $m_{c}=0.28 m_{0}$ are the density of states and electric conductivity effective masses for one of the six equivalent valleys, respectively; $m_{0}$ is the mass of a free electron.

The electron mobility in a nondegenerate semiconductor, limited by the elastic scattering on impurity ions (concentration $N_{i}$ and charge $\mathrm{Ze}$ ) according to $\mathrm{BH}$ model ${ }^{1-6}$ is

$$
\mu_{\mathrm{BH}}=\frac{2^{7 / 2}(4 \pi \epsilon)^{2}\left(k_{B} T\right)^{3 / 2}}{\pi^{3 / 2} e^{3} m^{1 / 2} Z^{2} N_{i}[\ln (1+\beta)-\beta /(1+\beta)]},
$$

where $\beta=(24 m \epsilon / n)\left(k_{B} T / e \hbar\right)^{2}$.

The electron mobility by the $\mathrm{CW}$ model ${ }^{4-6,10,25}$ is

$$
\mu_{\mathrm{CW}}=\mu_{\mathrm{BH}} \frac{\ln (1+\beta)-\beta /(1+\beta)}{\ln (1+\alpha)},
$$

where $\alpha=\left(4 \pi \epsilon /\left(Z e^{2}\right)\right)^{2}\left(3 k_{B} T\right)^{2} / N_{i}^{2 / 3}$ and $\mu_{\mathrm{BH}}$ is defined by formula (9).

\section{COMPARISON OF THE CALCULATIONS WITH EXPERIMENTAL DATA}

The calculation of the electron mobility in nondegenerate gas of electrons $\mu$ will be carried out assuming, that $\mu^{-1}=\mu_{1}^{-1}+\mu_{i}^{-1}$, where $\mu_{1}$ is the phonon scattering limited electron mobility in the crystal, and $\mu_{i}$ is the impurity ion scattering limited mobility.

Different experimental data ${ }^{26-30}$ on the dependence of the mobility of electrons on their concentration $n=(1$ $-K) N$ at $T=77-80 \mathrm{~K}$ in $n$ InSb crystals with average compensation ratio $K \approx 0.15$ is shown in Fig. 1 . Here, $K$ is the ratio of concentration of totally ionized hydrogenlike acceptors $(Z=-1)$ to donors $(Z=+1) ; N_{i}=(1+K) N$ is the concentration of impurity ions and $N$ is the concentration of

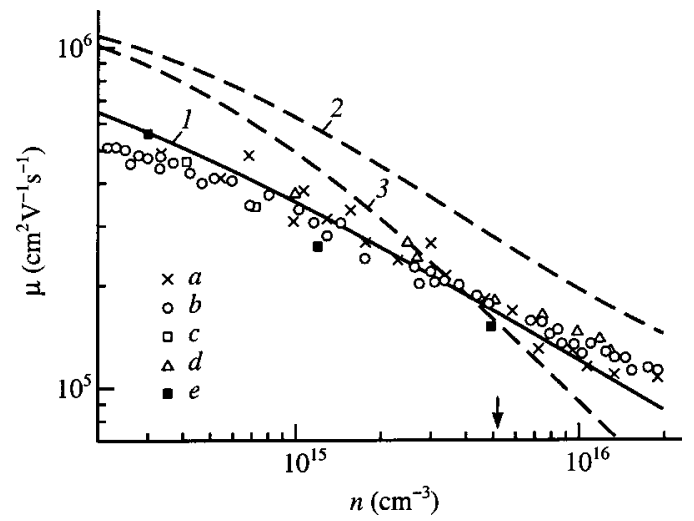

FIG. 1. The mobility $\mu$ of conduction electrons in $n$ InSb vs their concentration $n$ for $K \approx 0.15$ at $T=78 \mathrm{~K}$. The results of calculation of $\mu: 1$ using Eq. (8), when $|Z|=1$ and $\mu_{1}=1.5 \times 10^{6} \mathrm{~cm}^{2} \mathrm{~V}^{-1} \mathrm{~s}^{-1} ; 2$ using the BH formula (9); 3 using the CW formula (10). Experimental data on Hall mobility: [(a) see Ref. 26, (b) see Ref. 27, (c) see Ref. 28, and (d) see Ref. 29. Drift mobility (e) see Ref. 30]. The arrow shows effective density of states $N_{c}$ $=2\left(2 \pi m k_{B} T\right)^{3 / 2} /(2 \pi \hbar)^{3}$ in the $c$ band of InSb; $n \leqslant N_{c}$ corresponds to nondegenerate case.

donors. The mobilities of electrons (when $\mu_{1}$ was estimated by Refs. 7 and 26 as $\left.1.5 \times 10^{6} \mathrm{~cm}^{2} \mathrm{~V}^{-1} \mathrm{~s}^{-1}\right)$ were calculated using formulas (9), (10), and (8) with the following parameters: $\epsilon_{r}=17.8$ and $m=0.013 m_{0} \cdot{ }^{31}$ The arrow in Fig. 1 indicates the concentration $n=N_{c} \equiv 2\left(2 \pi m k_{B} T\right)^{3 / 2} /(2 \pi \hbar)^{3}$ of conduction electrons, above which their degeneration begins.

As seen in Fig. 1, formula (8) describes experimental data on mobility over a wide range of majority carrier concentrations. The condition $k_{t} R>1$, where $\hbar k_{t}=\left(3 m k_{B} T\right)^{1 / 2}$ is thermal quasimomentum of electrons, $R=(4 \pi(1$ $+K) N) / 3)^{-1 / 3}$, is satisfied. Note, that the BH (9) and CW (10) formulas give overestimated values of mobility. It should be noted, that for $n$ InSb crystals in Fig. 1, the density-of-states effective mass of electrons does not depend on their concentration. ${ }^{31}$ Note, that our calculations for other nondegenerate semiconductors with isotropic effective masses of conduction electrons also confirm the conclusions of papers (Refs. 7-9 and 12).

Thus, the model of majority charge carrier mobility, limited by elastic scattering on randomly distributed in crystal ionized impurities, is presented. The finiteness of electron (hole) interaction time with an ion and impact parameter limitations are considered simultaneously. It is shown, that the mobility of charge carriers in a crystalline semiconductor, calculated using our model agrees more closely to experimental data over a wide range of concentrations of the doping impurities and temperatures, than BH (9) and CW (10) formulas.

${ }^{1}$ H. Brooks, Phys. Rev. 83, 879 (1951).

${ }^{2}$ D. Chattopadhyay and H. J. Queisser, Rev. Mod. Phys. 53, 745 (1981).

${ }^{3}$ V. F. Gantmakher and I. B. Levinson, Current Carriers Scattering in Metals and Semiconductors (Nauka, Moscow, 1984) (in Russian).

${ }^{4}$ B. K. Ridley, Quantum Processes in Semiconductors (Clarendon, Oxford, 1999), Chap. 4.

${ }^{5}$ P. Y. Yu and M. Cardona, Fundamentals of Semiconductors: Physics and Materials Properties (Springer, Berlin, 1999), Chap. 5.

${ }^{6}$ K. Seeger, Semiconductor Physics (Springer, Berlin, 1999), Chap. 6.

${ }^{7}$ Y. M. Galperin, E. M. Gershenzon, I. L. Drichko, and L. B. Litvak- 
Gorskaya, Fiz. Tekh. Poluprovodn. (S.-Peterburg) 24, 3 (1990).

${ }^{8}$ J. R. Meyer and F. J. Bartoli, Phys. Rev. B 36, 5989 (1987).

${ }^{9}$ R. P. Joshi and D. K. Ferry, Semicond. Sci. Technol. 7, B319 (1992).

${ }^{10}$ E. Conwell and V. F. Weisskopf, Phys. Rev. 77, 388 (1950).

${ }^{11}$ J. M. M. Pantoja and J. L. S. Franco, IEEE Electron Device Lett. 18, 258 (1997).

${ }^{12}$ T. G. Van de Roer and F. P. Widdershoven, J. Appl. Phys. 59, 813 (1986).

${ }^{13}$ E. L. Nagaev, Zh. Eksp. Teor. Fiz. 100, 1297 (1991).

${ }^{14}$ L. D. Landau and E. M. Lifshitz, Quantum Mechanics (Nauka, Moscow, 1989), Chap. 6 (in Russian).

${ }^{15}$ It should be noted, that if a homogeneous flux of electrons falls onto a sphere of radius $R$, then the mean length of the electron trajectory inside the sphere without scattering is $4 R / 3$ and the mean passing time is $4 R /(3 v)$

${ }^{16}$ V. A. Nikerov and G. V. Sholin, Kinetics of Degradation Processes (Energoatomizdat, Moscow, 1985), pp. 31 and 32 (in Russian).

${ }^{17}$ L. D. Landau and E. M. Lifshitz, Mechanics (Nauka, Moscow, 1988) (in Russian).

${ }^{18}$ Here, we follow Ridley approximation [B. K. Ridley, Quantum Processes in Semiconductors (Clarendon, Oxford, 1999), Chap. 4] by excluding the "third body" from the scattering act.

${ }^{19}$ M. I. Dyakonov and A. V. Khaetskii, Zh. Eksp. Teor. Fiz. 99, 1066 (1991).

${ }^{20}$ Note, that according to R. Landauer and T. Martin, Rev. Mod. Phys. 66, 217 (1994) the tunneling time of particle with mass $m$ and magnitude $\hbar k$ of the imaginary momentum under opaque rectangular barrier of length $2 R$ is given by relation $2 R m / \hbar k$.

${ }^{21}$ When energy levels degenerate, then the perturbation theory is inapplicable to the perturbation, which lasts for a long time: Different degenerate intrinsic states entangle each other [L. D. Landau and E. M. Lifshitz, Quantum Mechanics (Izd. Nauka, Moscow, 1989), Chap. 6 (in Russian)].

${ }^{22}$ C. Hamaguchi, Basic Semiconductor Physics (Springer, Berlin, 2001), Chap. 6.

${ }^{23}$ M. V. Alekseenko, A. G. Zabrodskii, and L. M. Shterengas, Semiconductors 32, 720 (1998).

${ }^{24}$ M. Wenzel, G. Irmer, J. Monecke, and W. Siegel, Semicond. Sci. Technol. 13, 505 (1998).

${ }^{25}$ K. Somogyi, Diamond Relat. Mater. 11, 686 (2002).

${ }^{26}$ V. I. Petrovskii, N. N. Solov'ev, E. M. Omel'ianovskii, and V. S. Ivleva, Fiz. Tekh. Poluprovodn. (S.-Peterburg) 12, 1904 (1978).

${ }^{27}$ V. S. Ivleva, M. N. Kevorkov, R. S. Mitrofanova, A. N. Popkov, and V. I. Selyanina, Fiz. Tekh. Poluprovodn. (S.-Peterburg) 12, 534 (1978).

${ }^{28}$ F. Kuchar, E. Fantner, and G. Bauer, Phys. Status Solidi A 24, 513 (1974).

${ }^{29}$ R. T. Bate, R. D. Baxter, F. J. Reid, and A. C. Beer, J. Phys. Chem. Solids 26, 1205 (1965).

${ }^{30}$ L. E. Vorob'ev, M. M. Gnesin, and V. I. Stafeev, Fiz. Tekh. Poluprovodn. (S.-Peterburg) 7, 1982 (1973).

${ }^{31}$ Semiconductors-Basic Data, edited by O. Madelung (Springer, Berlin, 1996). 